\title{
Study and Modeling of Ferromagnetic Hysteresis
}

\author{
Monia Ferjani Jaafar \\ CEntre de REcherche en Productique (CEREP) \\ Ecole Nationale Supérieure d'Ingénieurs de Tunis \\ Université Tunis I \\ e-mail : Monia.Elferjani@esstt.rnu.tn
}

\author{
Mohamed Ali Jabri \\ CEntre de REcherche en Productique (CEREP) \\ Ecole Nationale Supérieure d'Ingénieurs de Tunis \\ Université Tunis I
}

\begin{abstract}
In this paper we are interested in the study and modeling of ferromagnetic hysteresis. We simulated the magnetic hysteresis based on the Jiles-Atherton (J-A) model that we have developed using Matlab-Simulink environment. The (J-A) model is based on physical considerations, it is characterized by five parameters and considered to our knowledge as the most accurate and complete one. It allows studying a wide range of ferromagnetic material hysteresis loops which justify in this work our choice for this model. In order to determine these five model parameters, we have to numerically analyze experimental data from the material hysteresis loop. For this purpose we applied the (J-A) model for the dynamic study of a magnetic circuit taking into account the phenomenon of hysteretic material.
\end{abstract}

Keywords-Ferromagnetic material; Hysteresis loop; Hysteresis modeling; Jiles- Atherton model; Magnetic circuit.

\section{INTRODUCTION}

For isotropic magnetic material, the following vectors: magnetic field $H$, the magnetic induction $B$ and the magnetization $M$ are always collinear; these three fundamental magnetic terms are related by the equation (1):

$$
B=\mu_{0}(M+H)
$$

where $\mu_{0}$ is the permeability of vacuum

However for a ferromagnetic material, the response to an excitation field $H$ depends also on the material magnetization history, indeed the relation between the excitation field $H$ and magnetization $M$ or induction $B$ is not linear.

The hysteresis curve in Fig.1 defines intrinsic characters of ferromagnetic material; it gives access to the material magnetic properties characterizing the hysteresis loop. We can enumerate: remanent induction $B_{r}$, saturation induction $B_{s}$, coercive field $H_{c}$ as well as hysteresis loss corresponding to the area enclosed by the hysteresis loop on the $B . H$ plane.

Considering the importance and the complexity of hysteresis loops measurements, the mathematical modeling of this phenomenon is necessary.

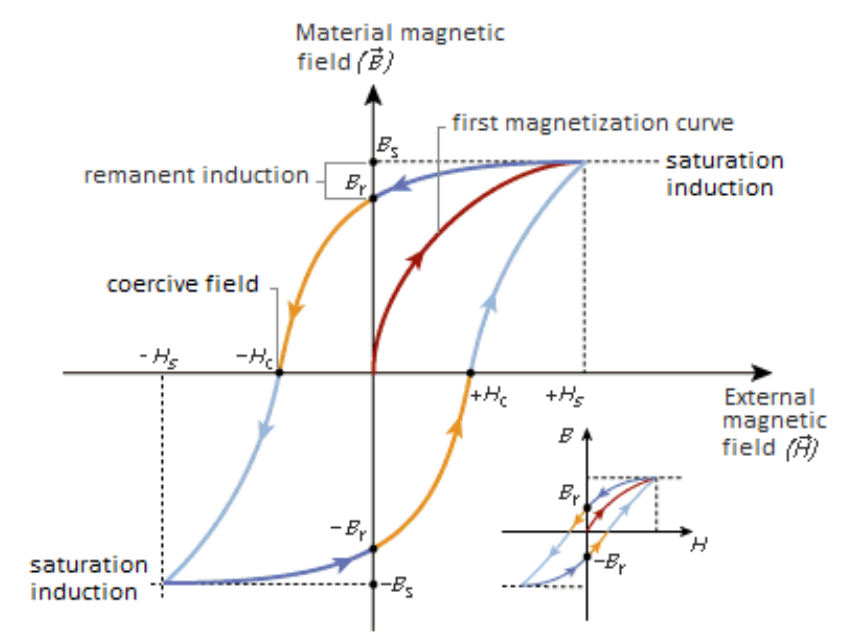

Fig. 1. Hysteresis loop of a ferromagnetic material

\section{MAGNETIC HYSTERESIS MODELS}

Hysteresis modeling and numerical analysis has a great importance in the domain of magnetic materials used in electrical engineering where the study of magnetic properties is essential.

There are several mathematical models describing the nonlinear characteristics of ferromagnetic materials. Many publications are devoted to this research topic: for example the analytical models which are based on mathematical modeling of the hysteresis phenomenon and the relation between magnetic induction and excitation field having polynomial or rational equation form, we can cite here the Stoner-Wohlfarth model and other models in the literature [1-10].

The improvement and optimization of these models should be developed in order to set up well defined approaches contributing to a better understanding of these hysteresis phenomena. Among these physical and mathematical methods one of the most reliable solutions is the Jiles-Atherton model which is considered as the most complete and precise one.

In this paper we set up a procedure developed under Matlab software package providing the simulation of the scalar ferromagnetic hysteresis using the Jiles Atherton model. We are also interested in applying this (J-A) model to a dynamic study of magnetic circuit. 


\section{JILES-ATHERTON MODEL}

Jiles and Atherton developed a mathematical model describing the nonlinear characteristics of magnetic materials. This model can determine the magnetic hysteresis $B-H$ curve. The theoretical background of the (J-A) model is the theory of Weiss and Langevin. This model is based on existing ideas of domain wall bending and translation. The assumption of this theory is the energy balance equation, and they suppose that energy exchange per volume unit is equal to the sum of magnetostatic energy exchange and hysteresis loss. Then magnetization is decomposed into two components, the reversible component representing the wall movement in the material, and the irreversible component corresponding hysteresis dissipated energy in the material $[1,2]$. The Jiles-Atherton model is based on physical considerations; it is defined by a simple first order differential equation and entirely characterized by five parameters.

For ferromagnetic materials, there are interactions between magnetic moments having an effective field $H_{e}$ defined by:

$$
H_{e}=H+\alpha M
$$

Where $\alpha$ the Weiss correction factor and represent the coupling between fields. For an ideal fer romagnetic material, the component $M_{a n}$ called anhysteretic curve which is the average of the ascending and the descending parts of the major hysteresis loop is given by the Langevin function

$$
M_{a n}\left(H_{e}\right)=M_{s a t}\left[\operatorname{coth}\left(\frac{H_{e}}{a}\right)-\left(\frac{a}{H_{e}}\right)\right]
$$

$M_{\text {sat }}$ is the saturation magnetization, and $a$ is the shape parameter of the anhysteretic curve.

For Jiles-Atherton model the magnetic moment per volume unit, i.e. the magnetization $M$ can be comprised of an irreversible component $M_{i r r}$ and a reversible component $M_{\text {rev }}$

$M=M_{r e v}+M_{i r r}$

$M_{r e v}$ is related to anhysteretic magnetization or ideal magnetization by the following expression:

$$
M_{\text {rev }}=c\left(M_{a n}-M_{i r r}\right)
$$

where $c$ is the reversibility coefficient depending on the material nature.

The irreversible component represents the irreversible displacement of the magnetic domain and which translates. Indeed, the differential susceptibility associated with the irreversible magnetization component is given by the expression (6) [1], $\frac{d M_{i r r}}{d H_{e}}=\frac{\left(M_{a n}-M_{i r r}\right)}{k \delta}$

where $k$ is the Boltzmann constant linked to the coercive field, the parameter $\delta$ is a factor which takes value +1 or -1 according to variation of excitation field $H$ by the time:

$$
\delta=\operatorname{sign}\left(\frac{d H}{d t}\right)
$$

By using the relations (4) and (5), the expression of magnetization is:

$$
M=(1-c) M_{i r r}+c M_{a n}
$$

After some mathematical formulation, the differential expression of magnetization can be written in this form:

$\frac{d M}{d H}=(1-c) \frac{\left(M_{a n}-M_{i r r}\right)}{\delta k-\alpha\left(M_{a n}-M_{i r r}\right)}+c \frac{d M_{a n}}{d H}$

The differential equations (9) describe magnetic hysteresis and it is characterized by five parameters.

TABLE I. PARAMETERS OF JILES ATHERTON MODEL

\begin{tabular}{|c|l|}
\hline$M_{s a t}$ & Saturation magnetization \\
\hline$\alpha$ & Domain interaction \\
\hline$a$ & Shape parameter of $\mathrm{M}_{\mathrm{an}}$ \\
\hline$c$ & Reversibility coefficient \\
\hline$k$ & Parameter linked to hysteresis loss \\
\hline
\end{tabular}

\section{A. Implementation of the model in Matlab environment}

The Jiles-Atherton model translating the magnetic hysteresis (described by the differential equation 9 and satisfying the equations 2 to 6 ), provide magnetization $M$ and magnetic induction $B$ starting from an applied excitation field $H$. We show in figure (2) the corresponding functional diagram for this scheme.

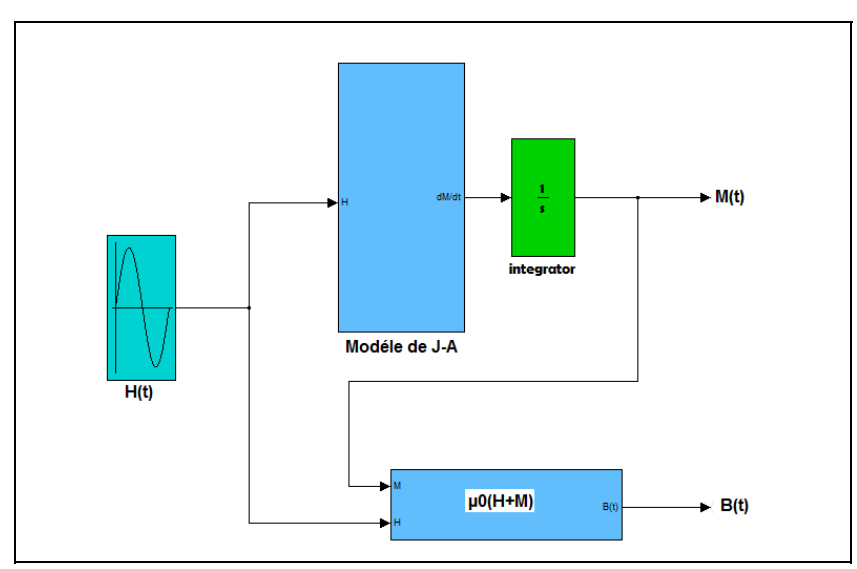

Fig. 2. Functional diagram of the Jiles-Atherton model. 


\section{B. Simulations results}

After numerical resolution of the differential equation describing the Jiles-Atherton model, we initially study the influence of the parameters $\left[M_{s a t}, \alpha, a, c, k\right]$ on the hysteresis loops obtained for purely sinusoidal excitation field, and then the hysteresis loops corresponding to various forms of excitations field $H$.

The effect of each parameter on the hysteresis loop shape is given respectively on figures $3,4,5,6$ and 7 .

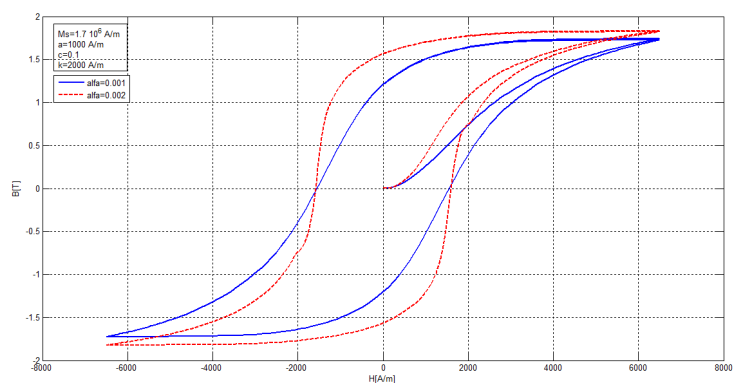

Fig. 3. $B$-H curve for two different values of $« \alpha »$

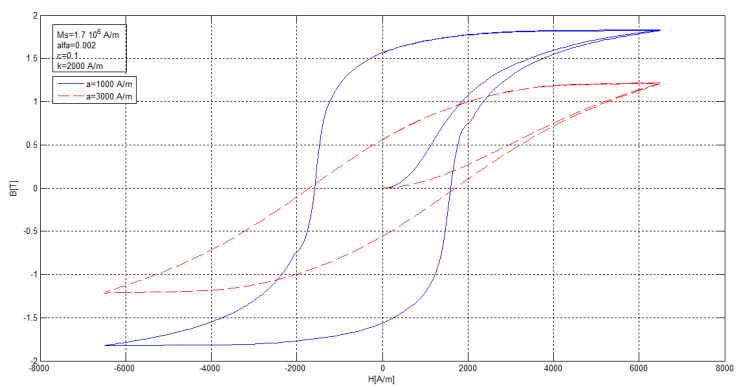

Fig. 4. $B$-H curve for two different values of « $a »$

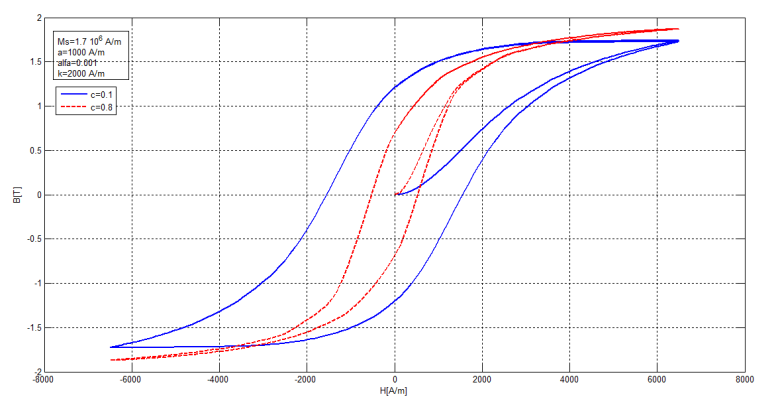

Fig. 5. $B$-H curve for two different values of $« c »$.

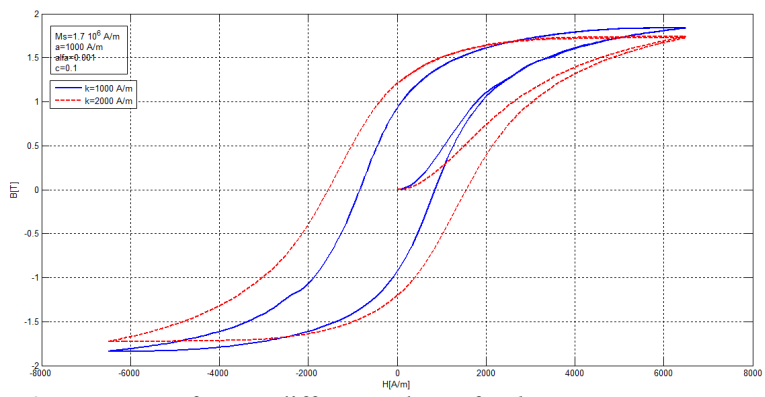

Fig. 6. $B$-H curve for two different values of « $k »$

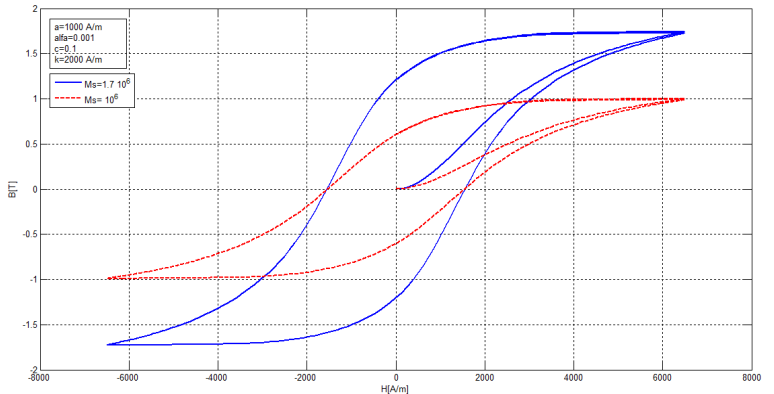

Fig. 7. $B$-H curve for two different values of « $\left.M_{\text {sat }}\right\rangle$

The five physical parameters of the Jiles-Atherton model make possible to define perfectly the hysteresis loops of ferromagnetic materials. Indeed, while varying each parameter of the model, we observe that the shape of hysteresis loops is changing.

So far, we study the effect of varying the excitation signal. We consider several excitation fields $H(t)$ respectively: perfect sinusoidal signal, imperfect sinusoidal signal having an alternate component in parallel with a continuous component, and finally an alternative signal having harmonics and a signal in form of damping sine The parameters of the model are:

$a=2000 \mathrm{~A} / \mathrm{m} ; \alpha=0.002 ; k=2000 \mathrm{~A} / \mathrm{m} ; c=0.1 ;$

$M_{\text {sat }}=1.7 .10^{6} \mathrm{~A} / \mathrm{m}$.

-Sinusoïdal excitation

$H(t)=H_{\max } \sin (\omega t)$, with $H_{\max }$ varying between $(2000$

and $10000 \mathrm{~A} / \mathrm{m})$.

Figure 8 represents the hysteresis loops and the corresponding $B(t)$ and $H(t)$ obtained for different amplitudes values of the sinusoidal excitation field for a 50 $\mathrm{Hz}$ frequency.

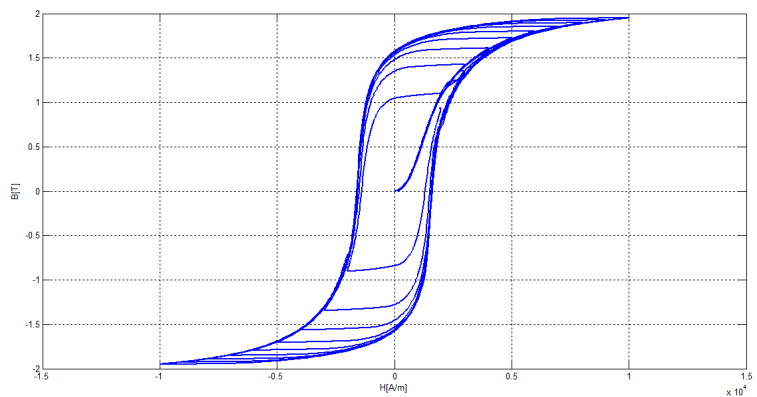

Fig. 8. B-H curve for excitations fields varying from $2000 \mathrm{~A} / \mathrm{m}$ to 10000 $\mathrm{A} / \mathrm{m}$.

- Sinusoidal excitation with a continuous component

$H(t)=H_{1} \sin (\omega t)+H_{2}$

$H_{1}$ is respectively $9000,7000,5500$ and $4500 \mathrm{~A} / \mathrm{m}$ and $H_{2}$ is respectively $0,2000,3500$ and $4500 \mathrm{~A} / \mathrm{m}$.

The hysteresis loops obtained are represented on figure 9. 


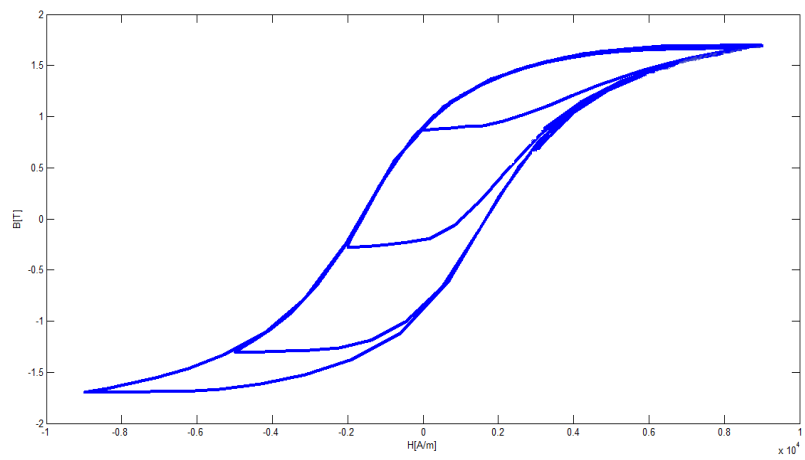

Fig. 9. Hysteresis loops corresponding to an excitation field with a continuous component.

- Sinusoidal excitation taking account of harmonic

Having in mind that electrical signals have many harmonics, obviously it is interesting to study this phenomenon applying a sinusoidal signal presenting a third order harmonic for example.

$H(t)=H_{1} \sin (\omega t)+H_{2} \sin (3 \omega t)$

-For $H_{1}=8000 \mathrm{~A} / \mathrm{m}$ and $H_{2}=1600 \mathrm{~A} / \mathrm{m}$, figure 10 represents the obtained hysteresis loop and the corresponding $H(t)$ and $B(t)$.

-For $H_{1}=8000 \mathrm{~A} / \mathrm{m}$ and $H_{2}=4800 \mathrm{~A} / \mathrm{m}$, figure 11 gives the obtained hysteresis loop and the corresponding $H(t)$ and $B(t)$.

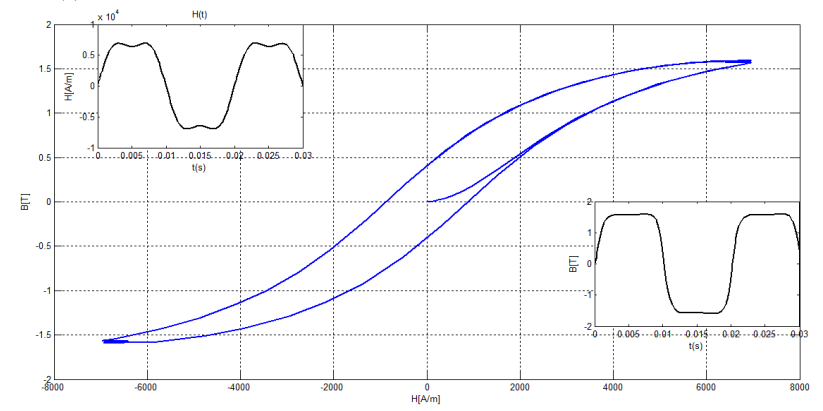

Fig. 10. Hysteresis loop and the corresponding $H(t)$ and $B(t)$.

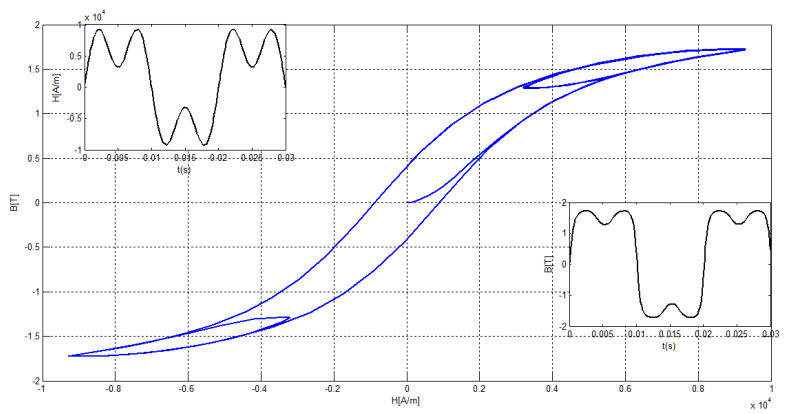

Fig. 11. B-H curve including minor loops and the corresponding $H(t)$ and $B(t)$.

-Damping sinusoidal excitation

To demagnetize ferromagnetic material we have to apply a damping sinusoidal excitation and we call it amortization signal:
$H(t)=H_{\max } \sin (\omega t) \cdot \exp \left(-\frac{t}{\tau}\right)$

Figure 12 represents the excitation field $H(t)$ and the induction field $B(t)$.
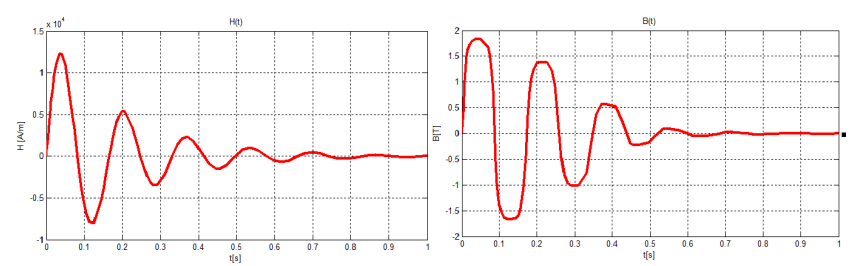

Fig. 12. Excitation field $\mathrm{H}(\mathrm{t})$ and the corresponding induction $\mathrm{B}(\mathrm{t})$

Figure 13 represents the hysteresis loops under an amortization signal of excitation.

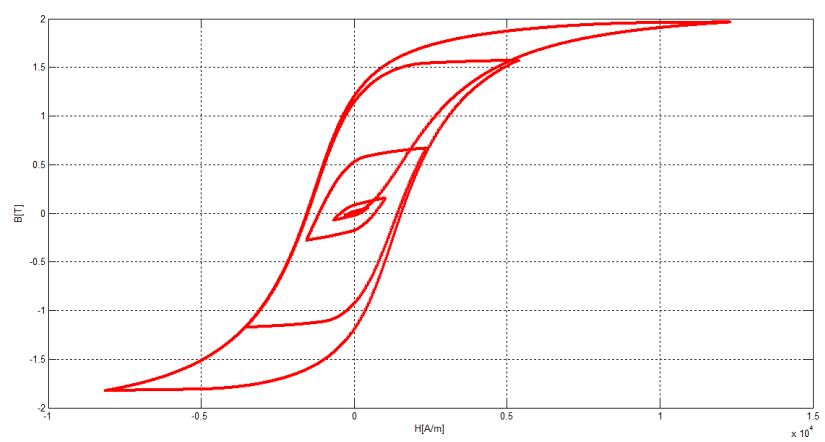

Fig. 13. Hysteresis loop under a decreasing amortization signal

\section{Interpretations}

Using the Jiles-Atherton model we are able to determine the hysteresis loop of a large range of ferromagnetic materials. Indeed, while varying the parameters of the model the hysteresis loop is modified. Thus the nature of excitation signal influences directly the shape of the hysteresis loop.

\section{DYNAMIC STUDY OF NON LINEAR MAGNETIC CIRCUIT INCLUDING HYSTERESIS}

We presented in this part a hysteretic modeling and a numerical simulation of a nonlinear magnetic circuit. The resolution of the differential equation translating the iron core model is carried out by applying the Jiles-Atherton model. We are interested in a RL circuit, including a linear resistor in series with a saturable inductor core and supplied with a sinusoidal voltage.

We consider a magnetic circuit without air-gap in toroidal shape core represented in figure 14. We suppose that the material is homogeneous and isotropic.

This torus carries a primary winding uniformly distributed, having $\mathrm{N}$ turns and a cross section $\mathrm{S}$ and which constitutes practically a flux tube. $\mathrm{d}_{1}$ and $\mathrm{d}_{2}$ are respectively the corresponding diameters, at the interior and external torus edge. We suppose that the torus thickness $h$ is very small compared to its average diameter. The primary winding is traversed by a current $\mathrm{i}(\mathrm{t})$.

We suppose that magnetic induction is uniform, constant and orthogonal with each section of the magnetic core. The expression of the magnetic flux is: 
$\phi(t)=B(t) \cdot S$

Taking into account the flux gap and applying the Faraday's law, the general equation of the terminal voltage around the coil is given by the equation (15). The equivalent model circuit is represented in the figure 14 .

$$
\mathrm{V}(\mathrm{t})=\mathrm{R} \cdot i(t)+\mathrm{L}_{0} \cdot \frac{d i(t)}{d t}+L_{1} \cdot i(t) \cdot \frac{d i(t)}{d t}
$$

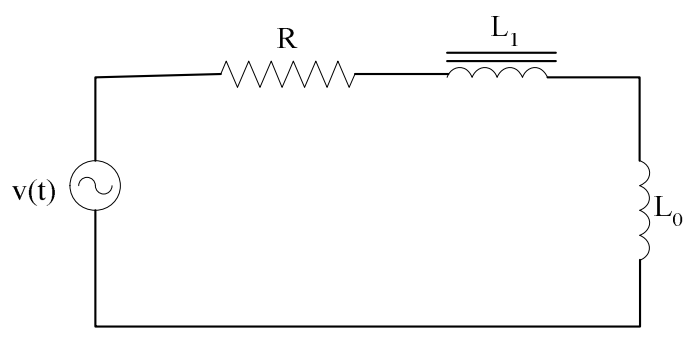

Fig. 14. Equivalent circuit.

With:

$\mathrm{L}_{0}$ is the inductance leakage of the magnetic circuit

$\mathrm{L}_{1}$ the proper inductance.

$\mathrm{R}$ the internal resistance of the iron core.

We propose to deduce the expression of the current $i(t)$ in the circuit integrating the magnetic hysteresis described by J-A model.

The expression of the terminal voltage $v_{L}$ is given by:

$$
v_{L}=N \cdot S \cdot \frac{d B}{d t}
$$

Then voltage $v(t)$ is written as follows:

$$
v(t)=\mathrm{R} . \mathrm{i}(\mathrm{t})+N \cdot S \cdot \mu_{0} \cdot \frac{d H}{d t}\left(1+\frac{d M}{d H}\right)
$$

By applying the Ampere theorem, we obtain the following expression:

$$
\frac{d i}{d t}=\frac{2 \pi R}{N^{2} \cdot S \cdot \mu_{0}} \cdot \frac{v(t)-R \cdot i(t)}{1+\frac{d M}{d H}}
$$

Where $\frac{d M}{d H}$ is the differential magnetization expression given by J-A model and equation 9 describing the system shown in figure 14 and taking into account both saturation and magnetic hysteresis.

The block diagram translating this scheme is given in the following figure 15 .

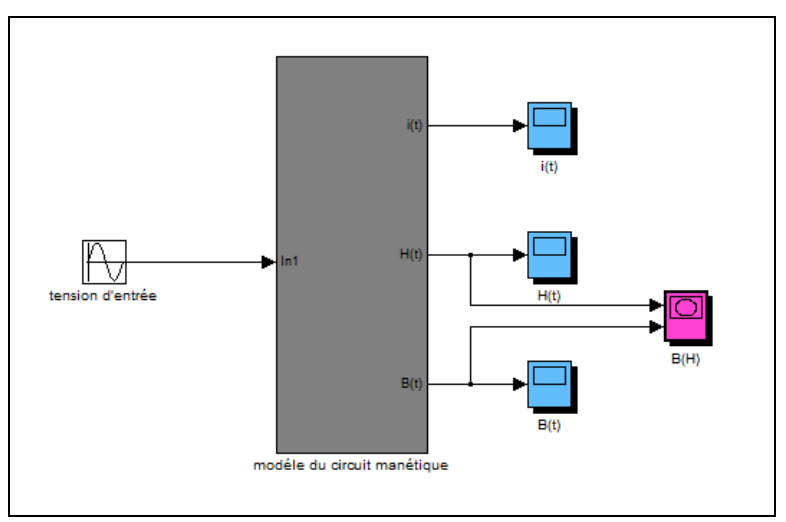

Fig. 15. Simulink bloc diagram of the dynamic's nonlinear circuit using the Jiles Atherton model.

The circuit is supplied by a sinusoidal alternative voltage, and the parameters of the circuit are given in table 2:

TABLE II. PARAMETERS OF THE CIRCUIT

\begin{tabular}{|c|c|}
\hline Parameter & Value \\
\hline $\mathrm{R}$ & $5 \Omega$ \\
\hline $\mathrm{D}$ & $0.1 \mathrm{~m}$ \\
\hline $\mathrm{S}$ & $510^{-3} \mathrm{~m}^{2}$ \\
\hline $\mathrm{N}$ & 50 turns \\
\hline
\end{tabular}

The simulations results from the Simulink model are given in figures $16,17,18,19,20$ and 21 where we respectively represent the magnetic induction $\mathrm{B}(\mathrm{t})$, the excitation $\mathrm{H}(\mathrm{t})$ and the corresponding hysteresis loop.

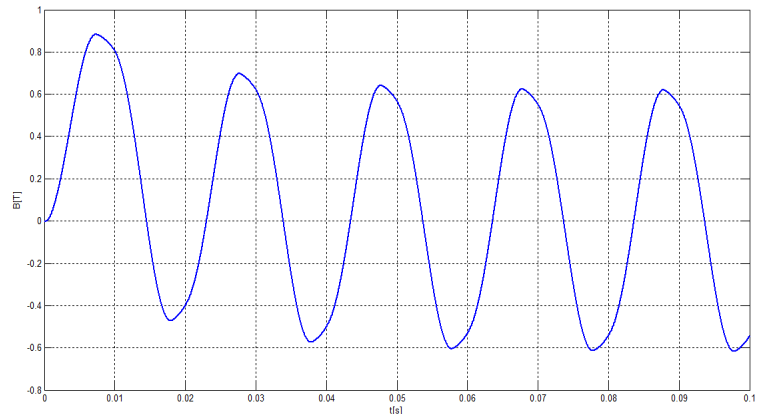

Fig. 16. Curve $B(t)$ for $\mathrm{V}_{\max }=100 \mathrm{~V}$

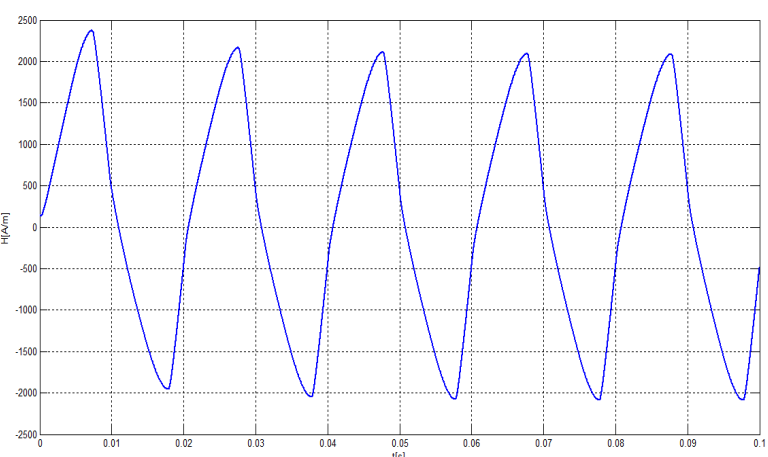

Fig. 17. Curve $H(t)$ for $\mathrm{V}_{\max }=100 \mathrm{~V}$. 


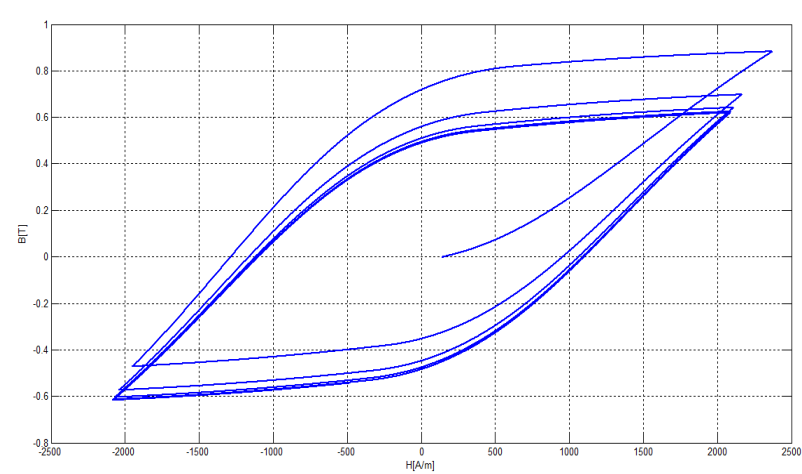

Fig. 18. Characteristic $B(H)$ for $\mathrm{V}_{\max }=100 \mathrm{~V}$.

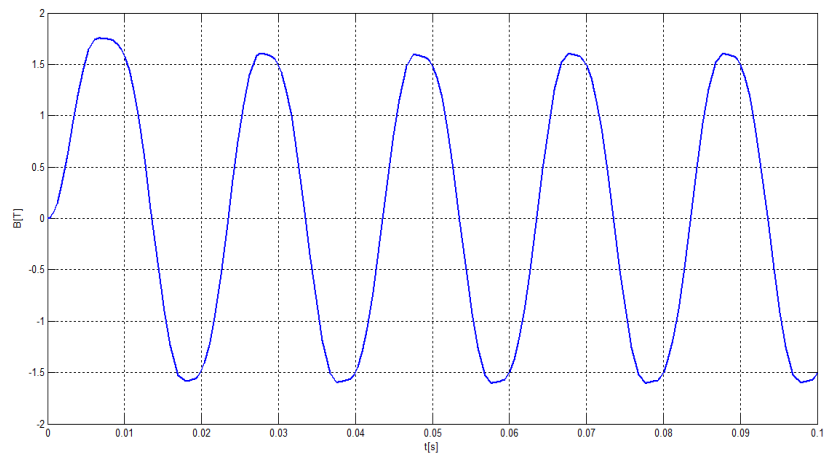

Fig. 19. Curve of $B(t)$ for $\mathrm{V}_{\max }=230 \mathrm{~V}$.

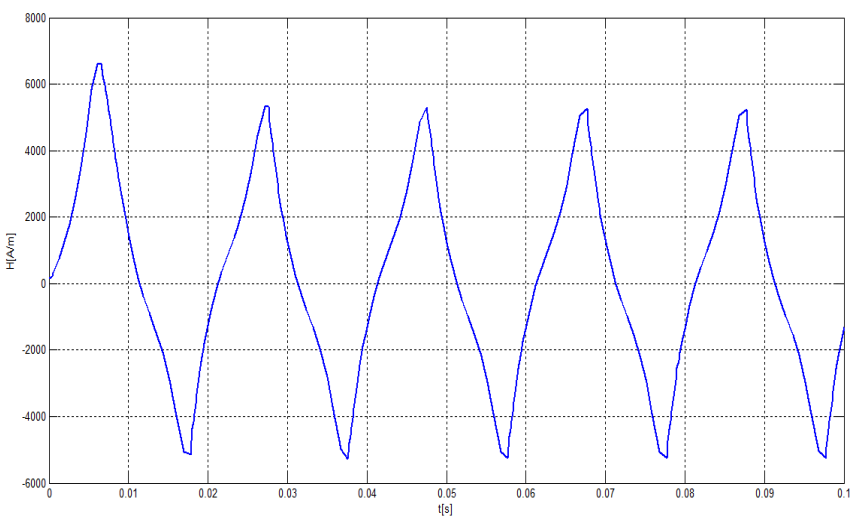

Fig. 20. Curve $H(t)$ for $\mathrm{V}_{\max }=230 \mathrm{~V}$.

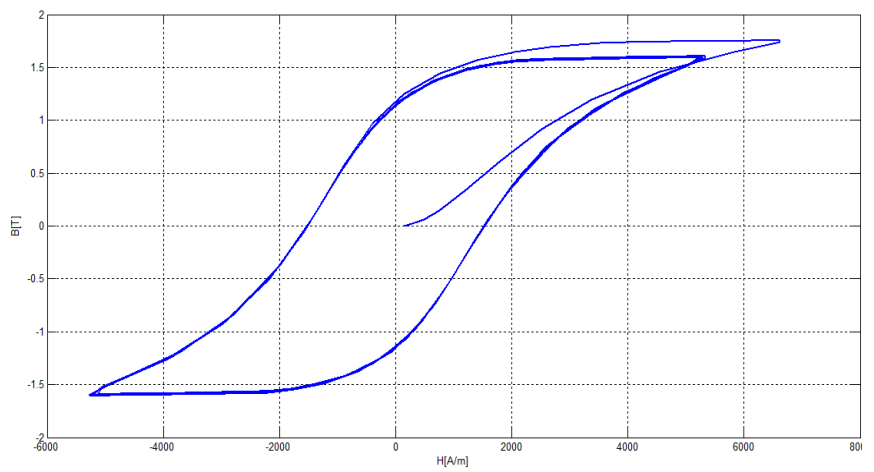

Fig. 21. Characteristic $B(H)$ for $\mathrm{V}_{\max }=230 \mathrm{~V}$.

\section{CONCLUSION}

We present in this paper an overview of some hysteresis models already existing in the literature. We decide to choose the Jiles-Atherton model developed by physical and mathematical approach of magnetic hysteresis phenomenon for ferromagnetic materials.

After setting up the mathematical improvement of the J-A model, we implement the later in the Matlab-Simulink software package. Through this procedure we are able to study the different aspects of hysteresis parameters and the model performance. Finally, we applied this model to the dynamic study of a nonlinear magnetic circuit including hysteresis phenomenon.

\section{REFERENCES}

[1] D.C. Jiles and J.L. Atherton, " Theory of ferromagnetic hysteresis », Journal of Magnetism and Magnetic Materials, Vol. 61, pp. 48-60, 1986.

[2] D.C. Jiles, Introduction to Magnetism and Magnetic Materials, Chapman \& Hall, London, 1991.

[3] R.M. Bozorth, "Ferromagnetism », IEEE Press Classic Reissue, New York, 1993.

[4] F.Preisach, «Uber die magnetische nachwirfung ,zeischrift fur physic» Vol .94,pp 277-302,1935.

[5] K. Chwastek and J. Szczygłowski, «An alternative method to estimate the parameters of Jiles-Atherton model», J. Magn. Magn. Mater. 314, pp. 47-51, 2007.

[6] F.Liozro, B.Helps, D.L. Atherton, « Macroscopic models of magnetization », IEEE. Trans. Magn. Vol. 36, pp 418-428, March 2000 .

[7] G. Bertotti, «Hysteresis in Magnetism », Academic Press, San Diego, 1998

[8] I D Mayergoyz « Mathematical Models of Hysteresis », New York: Springer, 1991

[9] Ivanyi A Hysteresis Models in Electromagnetic Computation Budapest: Akad'emiai Kiado, 1997

[10] H. Li, Q. Li, X-B Xu, T. Lu, J. Zhang, L. Li, “A Modified Method for Jiles-Atherton Hysteresis Model and Its Application in Numerical Simulation of Devices Involving Magnetic Materials", IEEE Transactions on Magnetics, vol.47, n5, pp.1094-1097, 2011. 